\title{
Peroxynitrite Activates the NLRP3 Inflammasome Cascade in SOD1(G93A) Mouse Model of Amyotrophic Lateral Sclerosis
}

Ilaria Bellezza, $1, *$

Email ilaria.bellezza@unipg.it AQ1

Silvia Grottelli, 1

Egidia Costanzi, 1

Paolo Scarpelli, 1

Eva Pigna, 2

Giulio Morozzi, 1

Letizia Mezzasoma, 1

Matthew J. Peirce, 1

Viviana Moresi, ${ }^{2}$

Sergio Adamo, 2

Alba Minelli, 1

1 Department of Experimental Medicine, P.le Severi AQ2

, University of Perugia, 06132 Perugia, Italy

2 DAHFMO Unit of Histology and Medical Embryology, Interuniversity Institute of Myology, Sapienza University of Rome, Rome, Italy in press Mol Neurobiol, 2017, PMID: 28357805

doi: $10.1007 / s 12035-017-0502-x$ 
NOX2

Caspase 1

Il-1 $\beta$

\section{Electronic supplementary material}

The online version of this article (doi: 10.1007/s12035-017-0502-x ) contains supplementary material, which is available to authorized users.

\section{Introduction}

\section{AQ3}

Amyotrophic lateral sclerosis (ALS) is a neurodegenerative disease characterized by a fast-progressing motor neuron death. Usually, patients show severe muscle atrophy, paralysis and ultimately denervation of respiratory muscles and surrender to the disease within 2-5 years from the clinical onset [1]. Most of the ALS cases are classified as sporadic, since there is no family history of the disease. The remaining few cases (5-10\%) are defined as familial since inherited in an autosomal dominant fashion. These two forms of ALS are clinically indistinguishable, notwithstanding the genetic differences. Almost $20 \%$ of familial cases are connected with mutations in the superoxide dismutase 1 (SOD1) gene [2], such as the substitution of glycine to alanine in position 93 (G93A). hSOD1(G93A) transgenic mice, which undergo a gradually progressive disease, are used as the gold standard in ALS research.

Non-cell autonomous mechanisms involving non-neuronal cells contribute to motor neuron death [3], suggesting that multiple damages, occurring in multiple cellular compartments, can ultimately lead to the neuromuscular failure, the main feature of ALS.

The reduction of mutant SOD1 in microglia extends survival in transgenic SOD1(G37R) mice and supports the hypothesis of the contribution of non-neuronal cell toxicity to the pathogenesis of the disease [4]. Therefore, it is accepted that the microglial compartment is one of the non-cell autonomous player in the killing of motor neurons $[5]$.

Indeed, the appearance of activated microglial and astroglial cells, a process known as neuroinflammation, characterizes ALS. Furthermore, in transgenic mouse models of mutant SOD1-associated familial ALS, the loss of motor neurons follows the appearance of activated microglial cells $[6,7]$, thus underpinning the fundamental role of microglial cells in the phenotype development.

Microglia are macrophage-like cells performing the main active immune defense towards infection or injury in the central nervous system (CNS). Because of these functions, microglia display a range of pattern recognition receptors (PRRs) and produce a few important pro-inflammatory mediators, such as tumor necrosis factor (TNF) $\alpha$ and interleukin $1 \beta$ (IL-1 $\beta$ ) [6], thus taking part in the inflammatory responses to stimuli. Since ALS pathogenesis is underpinned by a vicious cycle of inflammation and neurodegeneration, the ability of microglial cells to trigger and maintain chronic inflammation has strengthen its pivotal role in the disease $[6,7,8]$. For instance, in the CNS of ALS patients and mutant SOD1 transgenic mice, elevated levels of IL-1 $\beta$ were found [9]. However, it is still unclear the cellular source of this IL- $1 \beta$ or the stimulus that drives its expression. IL-1 $\beta$ is expressed as an inactive precursor which, to be activated, has to undergo a proteolytic processing by caspase1. Caspase1, in turn, is bound to and activated by a multiprotein complex, to form the inflammasome. A range of substances that arise during infections, tissue damage or metabolic imbalance causes the inflammasome formation.

Commonly, inflammasomes are composed by three elements: a cytosolic PRR, pro-caspase1 and a PRR-specific adaptor molecule. The best-characterized inflammasome forming PRR is NOD-like receptor pyrin domain containing three (NLRP3) [ 10, 11]. NLRP3, activated by a wide range of molecules related to damage or infection, oligomerizes with the adaptor ASC (apoptosis-associated speck-like protein containing a caspase recruitment domain) and caspase 1 to form the NLRP3-inflammasome, which leads to the processing of pro- to mature IL-1 $\beta$ and its release. Although the likely importance of IL-1 $\beta$ in ALS and the potential capacity of NLRP3 to associate inflammation to IL- $1 \beta$ production, relatively few studies have surveyed the link between ALS and NLRP3 $[12,13,14,15]$.

In this study, we investigated the inflammasome activation in the spinal cord of hSOD1(G93A) mice and in murine 
microglial cells overexpressing hSOD1(G93A) and hypothesized that, by activating NLRP3 in glial cells, inflammation-induced peroxynitrite could drive chronic inflammation in the context of ALS.

\section{Materials and Methods}

\section{Materials}

All the reagents, unless otherwise stated, were from Sigma-Aldrich (St. Louis, MO). All the antibodies, unless otherwise stated, were from Santa Cruz Biotech (Santa Cruz, CA). Cell culture reagents were from Life Technologies (GibcoBRL, Gaithersburg, MD).

Mice

Thirteen-week-old (W13) female C57BL/6 and SOD1(G93A) mice were treated in strict accordance to the guidelines of the Institutional Animal Care and Use Committee and to National and European legislation, throughout the experiments. LPS (5 mg/kg) was administered i.p., and animals were killed after $24 \mathrm{~h}$.

\section{Immortalized Microglia}

Immortalized microglial cells, obtained from embryonic (E14) cortices from non-transgenic and transgenic hSOD1(G93A) mice following the method described by Righi and colleagues [16], were a kind gift of Dr. G. Pietrini (Università di Milano). Microglia were characterized by Western Blot and immunofluorescence for the presence of selective markers (CSF-1) and the absence of astrocyte-specific molecules (i.e., glial fibrillary acidic protein).

\section{Cell Cultures and Treatments}

Non-transgenic and SOD1(G93A) microglial cells were cultured in DMEM F-12 supplemented with 5\% fetal bovine serum (FCS), glutamine $(4 \mathrm{mM})$, penicillin $(50 \mathrm{U} / \mathrm{ml})$ and streptomycin $(50 \mathrm{mg} / \mathrm{ml})$ at $37{ }^{\circ} \mathrm{C}$ in a humidified $5 \% \mathrm{CO}_{2}$ environment and seeded at the density of $31.00094,000 / \mathrm{cm}^{2}$. After a $24-\mathrm{h}$ subculture, cells were exposed to $1 \mu \mathrm{g} / \mathrm{ml}$ LPS for various time. When inhibitors were used, $1400 \mathrm{~W}$ (iNOS inhibitor) and apocynin (APO, NOX2 inhibitor), were added to SOD1(G93A) cells $30 \mathrm{~min}$ prior to $1 \mu \mathrm{g} / \mathrm{ml}$ LPS exposure. AQ4

\section{Measurement of NO Production}

Nitric oxide (NO) production was determined indirectly through the measurement of nitrite, a stable metabolite of nitric oxide, by Griess reaction [17]. Results were expressed as percentage of the control, assumed as $100 \%$. Nitrite standard reference curve was prepared for each determination.

\section{Measurement of Cytokine Levels by ELISA}

Cytokine production was measured with an enzyme-linked immunosorbent assay (ELISA) kit in cell medium collected from control and LPS-treated cells. Samples were analyzed according to the manufacturer' s protocols (BD Biosciences Pharmigen, San Diego, CA).

\section{RT-PCR}

Total RNA was isolated with TRIZOL Reagent (Invitrogen Ltd., Paisley, UK) according to the manufacturer's instructions, and complementary DNA (cDNA) was synthesized using iScript cDNA synthesis kit (Bio-Rad Lab, Hercules, CA). PCR products from synthetized cDNA were amplifield with specific mouse primer sequences obtained from Invitrogen (Invitrogen Ltd., Paisley, UK) and listed in Table 1. Two microliters of cDNA was used for each PCR reaction using Taq DNA polymerase (New England Biolabs, Whitby, ON, Canada), according to the manufacturer's protocol. The products were visualized on ethidium bromide-stained $1.8 \%$ agarose gels.

\section{Table 1}

List of primers

AQ5

Gene name

Glyceraldehyde-3-phosphate-dehydrogenase
Gene symbol

Gapdh
Primer sequences (F: forward; $R$ : reverse)

F: GCCAAATTCAACGGCACAGT 


\begin{tabular}{|c|c|c|}
\hline & & \multirow{2}{*}{ R: AGATGGTGATGGGCTTCCC } \\
\hline & & \\
\hline \multirow{2}{*}{ Interleukin 1beta } & \multirow{2}{*}{$I L 1 \beta$} & F: AAAAGCCTCGTGCTGTCGGACC \\
\hline & & R: TTGAGGCCCAAGGCCACAGGT \\
\hline \multirow{2}{*}{ Interleukin 6} & \multirow{2}{*}{ IL6 } & F: GCTGGAGTCACAGAAGGAGTGGC \\
\hline & & R: GGCATAACGCACTAGGTTTGCCG \\
\hline \multirow{2}{*}{ Tumor necrosis factor alpha } & \multirow{2}{*}{$\operatorname{Tnf} \alpha$} & F: GCCCACGTCGTAGCAAACCAC \\
\hline & & R: GGCTGGCACCACTAGTTGGTTGT \\
\hline \multirow{2}{*}{ NLR family, pyrin domain containing $1 \mathrm{~A}$} & \multirow{2}{*}{ Nlrpla } & F: CTTGAGAAGTTGGGTGGGGT \\
\hline & & R: GATGGAGCAACTCAGGACCA \\
\hline \multirow{2}{*}{ NLR family, pyrin domain containing 3} & \multirow{2}{*}{$N L R P 3$} & F: GACACGAGTCCTGGTGACTTT \\
\hline & & R: CAGACGTATCTGAGCCAT \\
\hline \multirow{2}{*}{ NLR family, CARD domain containing 4} & \multirow{2}{*}{$I P A F$} & F: TCAGGTCACAGAAGACCT \\
\hline & & R: TTCACCCAGGGGGTAGAAGT \\
\hline \multirow{2}{*}{ Absent in melanoma 2} & \multirow{2}{*}{$\operatorname{Aim} 2$} & F: AAAACTGCTCTGCTGCCTCT \\
\hline & & R: GATGGCTTCCTGTTCTGCCA \\
\hline \multirow{2}{*}{ PYD and CARD domain containing } & \multirow{2}{*}{ Asc } & F: AACTGCGAGAAGGCTATGGG \\
\hline & & R: TGAGCTCCAAGCCATACGAC \\
\hline \multirow{2}{*}{ Heme-binding membrane glycoprotein gp91phox } & \multirow{2}{*}{ gp91phox } & F: TCACCACTAGTACCAGCATCACCA \\
\hline & & R: ACTCTGTCTTGCATTTCTGGATGCC \\
\hline \multirow{2}{*}{ Inducible nitric oxide synthase } & \multirow{2}{*}{$i N O S$} & F: GGTGTTCTTTGCTTCCATGCTAAT \\
\hline & & R: GTCCCTGGCTAGTGCTTCAGA \\
\hline
\end{tabular}

\section{Real-Time PCR}

Total RNA was isolated with TRIZOL Reagent (Invitrogen Ltd., Paisley, UK) according to the manufacturer's instructions, and cDNA was synthesized using iScript cDNA synthesis kit (Bio-Rad Lab, Hercules, CA). Real-time PCR was performed using the iCycler iQ detection system (Bio-Rad) and SYBR Green chemistry. Mouse primer sequences, obtained from Invitrogen (Invitrogen Ltd., Paisley, UK) were listed in Table 1. SYBR Green RT-PCR amplifications were carried out in a 96-well plate in a $25-\mu 1$ reaction volume that contained $12.5 \mu 1$ of $2 \times \mathrm{iQ}^{\mathrm{TM}}$ SYBR ${ }^{\circledR}$ Green SuperMix (Bio-Rad), $400 \mathrm{nM}$ forward and reverse primers and 5 to $40 \mathrm{ng}$ of cDNA. In each assay, no-template controls were included and each sample was run in triplicates. The thermal profile consisted of incubation at $95{ }^{\circ} \mathrm{C} 3 \mathrm{~min}$, followed by 40 cycles of denaturation for $10 \mathrm{~s}$ at $95{ }^{\circ} \mathrm{C}$ and an annealing/extension step of $30 \mathrm{~s}$ at $62^{\circ} \mathrm{C}$. Mean of $\mathrm{Ct}$ values of the stimulated sample was compared with the untreated control sample. $\Delta \mathrm{Ct}$ is the difference in $\mathrm{Ct}$ values derived from the target gene (in each assayed sample) and Gpdh, while $\Delta \Delta \mathrm{Ct}$ represents the difference between the paired samples. The $n$-fold differential ratio was expressed as $2^{-\Delta \Delta \mathrm{Ct}}$.

\section{Western Blotting Analyses}

Non-transgenic and SOD1(G93A) total cell lysate were obtained using hot Laemli sample buffer $1 \times(60 \mathrm{mM}$ Tris $\mathrm{HCl}, \mathrm{pH}$ 6.8, 2\% SDS, 10\% glycerol, $0.72 \mathrm{M} \beta$-mercaptoethanol). Extracts were loaded on SDS-polyacrylamide gel, transferred on nitrocellulose membrane and immunoblotted with phospho-NF-kB p65 (Ser536) antibody (1:1000) (Cell Signaling Technology, Danvers, MA), NLRP3 (Cryo-2) antibody (1:1000; AdipoGen Corp., San Diego, CA), iNOS (M-19) antibody (1:200), caspase1 antibody (1:1500; Cell Signaling Technology, Danvers, MA), gp91 phox (BD Biosciences Pharmigen, San Diego, CA) antibody (1:2000), IL-1 $\beta$ (1:250; Cell Signaling Technology, Danvers, MA) and horseradish peroxidase-conjugated anti-IgG antibody (1:5000). GAPDH (6-C5; 1:500) actin (I-19; 1:400) antibodies were used as marker protein for total extracts and membrane extracts. Immunocomplexes were visualized with an enhanced chemiluminescence kit (ECL, Pierce Biotechnology, Rockford, IL).

\section{Caspase1 Activity}


Cell pellet from SOD1(G93A) cells were treated with lysis buffer (50 mM HEPES, pH 7.4, 0.1\% CHAPS, $0.1 \mathrm{mM}$ EDTA) and incubated $10 \mathrm{~min}$ on ice. After centrifugation, protein extract $(50 \mu \mathrm{g})$ was incubated with a caspase 1 reaction mixture (20 mM PIPES, pH 7.4, $30 \mathrm{mM} \mathrm{NaCl}, 0.1 \%$ CHAPS, $1 \mathrm{mM}$ EDTA, 10\% sucrose) containing $50 \mu \mathrm{M}$ caspase 1 fluorogenic peptide substrate (Ac-YVAD-AFC). Then, elicited fluorescence was recorded ( $\lambda$ exc $380 \mathrm{~nm}$ and $\lambda \mathrm{em} 505 \mathrm{~nm})$. The florescence of AFC (fluorocoumarin) standard solutions $(0-5 \mu \mathrm{M})$ was used to construct a calibration curve and caspase 1 activity was expressed as pmol of AFC released $/ \mathrm{min} / \mathrm{mg}$ protein.

\section{Measurement of Intracellular Fluorescence}

SOD1(G93A) microglial cells, seeded on glass coverslips, were used for evaluation of ROS production, detection of activate caspase 1, immunolocalization of nitrated protein and actin labeling. Lumbar spinal cords (L4-L6) were dissected, embedded in tissue freezing medium (Leica, Wetzlar, Germany) and frozen in isopentane pre-cooled in liquid nitrogen. Cryosections of $16 \mu \mathrm{m}$ thickness were obtained using a Leica cryostat and used to detect activate caspase1 and immunolocalization of nitrated protein.

ROS Production The 2',7'-dichlorodihydrofluorescein diacetate (DCFH-DA) method was used to detect ROS intracellular levels $[18,19]$. The fluorescence of $2^{\prime}, 7^{\prime}$-dichlorofluorescein was detected at $485 \mathrm{~nm}$ excitation and at $535 \mathrm{~nm}$ emission.

Detection of Activate caspase1 SOD1(G93A) cells were loaded with $50 \mu \mathrm{M}$ Ac-YVAD-AFC for $1 \mathrm{~h}$ at $37^{\circ} \mathrm{C}$ and fixed for $20 \mathrm{~min}$ in $4 \%$ PFA at room temperature.

Immunolocalization of Nitrated Protein Cells were fixed with 4\% PFA for 20 min at room temperature and incubated overnight at $4{ }^{\circ} \mathrm{C}$ with nitrotyrosine antibody (1:50; Sigma-Aldrich, St. Luis, MO).Cells were then incubated with tetramethylrhodamine (TRITC)-conjugated anti-rabbit IgG (1:100; Jackson ImmunoResearch Laboratories, INC, PA, USA) for $1 \mathrm{~h}$ at room temperature. Control samples were incubated with non-immune serum.

Actin Labeling Phalloidin was used to detect filamentous actin (F-actin) content on SOD1(G93A). The cells were fixed with 4\% PFA for $20 \mathrm{~min}$ at room temperature and F-actin was stained with tetramethylrhodamine TRITClabeled phalloidin $(1: 250)$ for $30 \mathrm{~min}$ at room temperature.

At the end of each experimental procedure, cell nuclei were counter-stained with 4',6-diamidino-2-phenylindole (DAPI) and samples were analyzed with a Zeiss Axio Observer Z1 equipped with Apotome and Digital Camera Axiocam MRm Zeiss.

\section{Immunoprecipitation}

SOD1(G93A) microglial cells were lysed in RIPA buffer (50 mM Tris HCl, pH 8.0, $150 \mathrm{mM} \mathrm{NaCl,} 1 \mathrm{mM}$ EDTA, $0.05 \%$ sodium deoxycholate, $1 \%$ Triton X-100, 0.1\% SDS, $1 \mathrm{mM}$ PMSF, protease inhibitors) and immunoprecipitated with nitrotyrosine antibody (1:200) and protein A sepharose. Immunoprecipitated complexes were washed in lysis buffer and boiled for $5 \mathrm{~min}$ in Laemli Sample Buffer $1 \times$. Samples were loaded on SDS-polyacrylamide gel, transferred on nitrocellulose membrane and immunoblotted with NLRP3 (Cryo-2) antibody $(1: 1000)$ and nitrotyrosine antibody $(1: 2000)$.

\section{Statistical Analysis}

All results were confirmed in at least three separate experiments and expressed as mean \pm SD. Data were analyzed for statistical significance by Student's $t$ test. $p$ values $<0.05$ were considered significant.

\section{Results}

\section{LPS Activates NLRP3 Inflammasome in SOD1(G93A) Microglial Cells}

Since microglial cells are the key players in neuroinflammatory reactions, to determine the mechanisms leading to neuroinflammation in ALS, we used immortalized microglial cells from hSOD1(G93A) mice. It has been reported that the exposure of BV2 microglial cells to exogenous SOD1(G93A)-induced activation of the inflammasome [12]. Therefore, we sought to determine whether the endogenous expression of SOD1(G93A) could induce the same effect. We first evaluated the expression of inflammasome components in murine immortalized hSOD1(G93A) overexpressing microglial cells (SOD1(G93A)) (Fig. 1a) and found that NLRP1, NLRP3, IPAF and AIM2 inflammasome receptors messenger RNAs (mRNAs) are expressed in SOD1(G93A) cells, together with the 
inflammasome adapter protein ASC, suggesting that, upon stimulation, cells can be able to activate the inflammasome. Therefore we exposed SOD1(G93A) microglial cells to $1 \mu \mathrm{g} / \mathrm{ml}$ LPS and evaluate the changes in gene expression of the inflammasome components (Fig. 1b) and found that a 3-h LPS exposure is sufficient to increase the expression of the sole NLRP3 inflammasome. This result has been confirmed on protein level (Fig. 1c). Indeed, a 6-h LPS exposure caused a robust increase of NLRP3 protein in SOD1(G93A) microglial cells. The observed increase in NLRP3 expression prompted us to evaluate inflammasome activation. We determined caspase1 activation in SOD1(G93A) microglial cells and found that a 6-h LPS exposure caused an increase in the cleaved form of caspase1 (Fig. 1d). To further analyze caspase1 activation in SOD1(G93A) cells, we used the fluorescent YVAD substrate and found that LPS induced an increase in caspase1-mediated substrate cleavage (Fig. 1e) corresponding to a doubling of caspase 1 specific activity (Fig. 1e). The addiction of ATP failed to further increase caspase 1 activity (data not shown). The activation of caspase 1 through inflammasome results in the secretion of mature IL-1 [11]. Indeed, we found that LPS exposure induces a significant increase in IL-1 $\beta$ secretion that is accompanied by an increase in IL-17 secretion (Fig. 1f), a direct target of IL-1 $\beta$ in microglial cells [20]. The presence of the cleaved form of IL-1 $\beta$ was confirmed by Western blotting (Fig. 1g). We did not observe any LPS-induced increase in caspase 1 activation in non-transgenic microglial cells (Fig. 1h). These results suggest that LPS is capable of activating a functional NLRP3 inflammasome in SOD1(G93A) microglial cells.

Fig. 1

LPS activates NLRP3 inflammasome in SOD1(G93A) microglial cells. Untreated SOD1(G93A) microglial cells were used for a RT-PCR analysis of inflammasome component expression (1, NLRP1; 2, NLRP3; 3, IPAF-1; 4, AIM2; 5, ASC); SOD1(G93A) microglial cells were exposed to $1 \mu \mathrm{g} / \mathrm{ml}$ LPS and used for $\mathbf{b}$ qRT-PCR of the indicated genes after a 3-h LPS exposure. Gene expression values were normalized to Gapdh and presented as $2^{-\Delta \Delta \mathrm{Ct}}$. Relative mRNA gene abundance in untreated cells was assumed to be 1 (control). Data represent mean $\pm \mathrm{SD}(n=3)$. ${ }^{*} p<0.05$ vs. untreated cells. $\mathbf{c}$ Western blotting analysis of NLRP3 and $\mathbf{d}$ active caspase 1 after $6 \mathrm{~h}$ LPS exposure. GAPDH was used as loading control. The images are representative of one out of three separate experiments; e detection of activated caspase1/specific enzyme activity assay after a 6-h LPS exposure. Scale bar, $100 \mu \mathrm{m}$; f cytokine secretion were assayed in cell medium by ELISA after an 18-h LPS exposure. Data represent mean $\pm \mathrm{SD}(n=3)$. ${ }^{*} p<0.05$ vs. untreated cells; g Western blotting analysis of IL-1 $\beta$. h Non-transgenic microglial cells were exposed to $1 \mu \mathrm{g} / \mathrm{ml} \mathrm{LPS}$ and used for Western blotting analysis of active caspase1. The images are representative of one out of three separate experiments. GAPDH was used as loading control

AQ6 
A
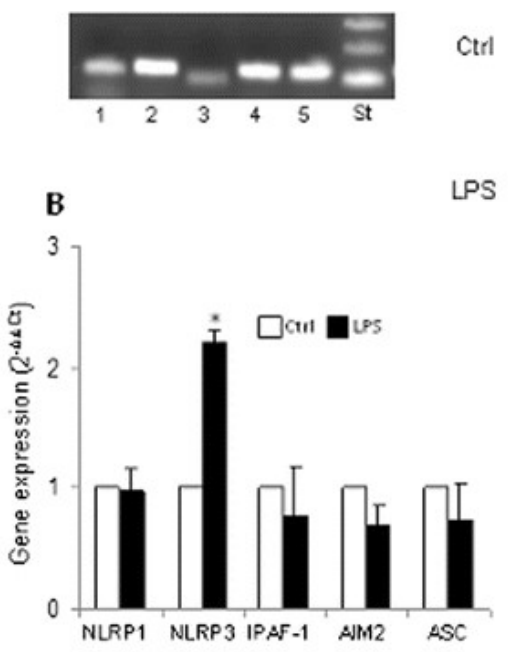

C.
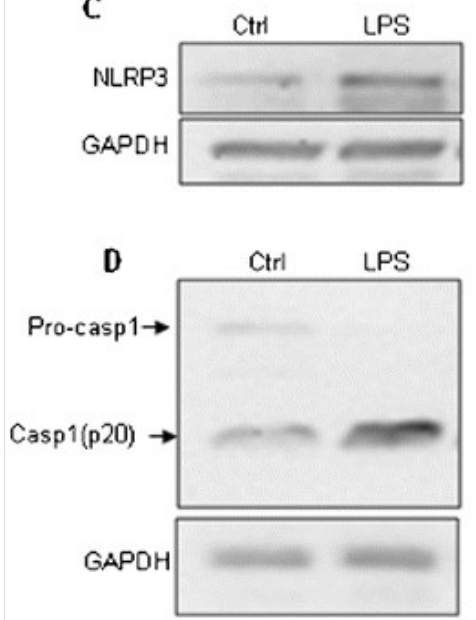
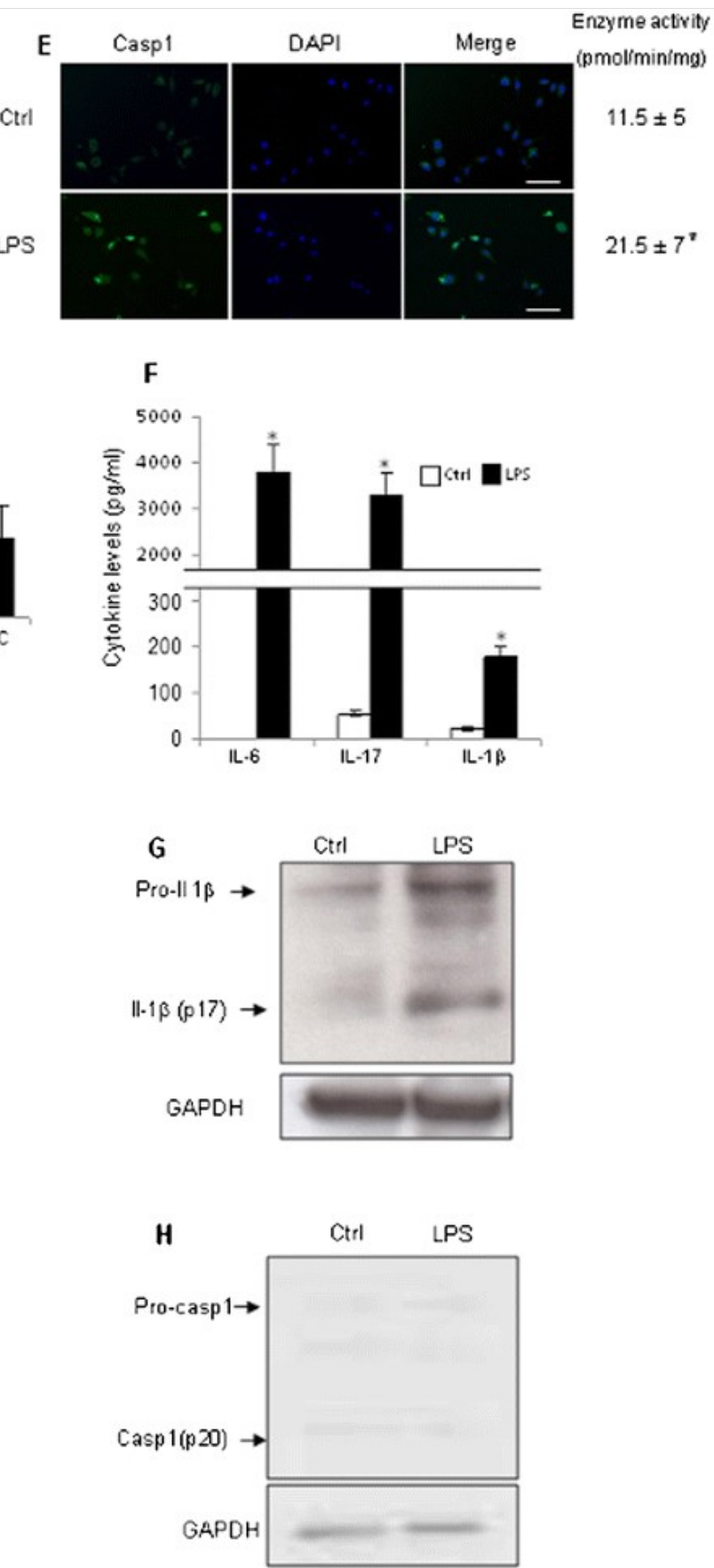

\section{LPS Induces Protein Nitration in SOD1(G93A) Microglial Cells}

LPS is a toll-like receptor 4 ligand known to induce an inflammatory response in microglial cells [21]. To test whether the canonical response is also activated in SOD1(G93A) microglial cells, we first determined the effects of LPS exposure on cell morphology and found that, in the presence of LPS, cells assume an amoeboid shape characteristic of microglial activation (Fig. 2a). Moreover, LPS induces the activation of the transcription factor $\mathrm{NF}-\kappa \mathrm{B}$ as detected by the increase in the phosphorylation/activation of the p65 subunit, already after a 3-h exposure (Fig. 2b). The activation results in an increase in the expression of several pro-inflammatory genes such as IL-6, IL-1 $\beta$, TNF $\alpha$, (Fig. 2c), inducible nitric oxide synthase (iNOS) and the gp91phox subunit of NADPH oxydase (NOX)2 (Fig. 2d, e). The gene expression parallels the increase in cytokine secretion (Fig. 1f) and the protein expression (Fig. 2d, e).

Fig. 2

LPS induces protein nitration in SOD1(G93A) microglial cells. SOD1(G93A) microglial cells were treated with $1 \mu \mathrm{g} / \mathrm{ml}$ LPS. a Phalloidin staining after a 24-h exposure. Scale bar, $100 \mu \mathrm{m}$; b time course of p-NF- $\kappa$ B. At each indicated time, cells were collected and total extract analyzed by Western blotting. GAPDH was used as loading control. The images are representative of one out of three separate experiments; $\mathbf{c}$ qRT-PCR of the indicated genes after a 6-h LPS exposure. Gene expression values were normalized to Gapdh and presented as $2^{-\Delta \Delta \mathrm{Ct}}$. Relative mRNA gene 
abundance in untreated cells was assumed to be 1 (control). Data represent mean $\pm \mathrm{SD}(n=4) .{ }^{*} p<0.05$ vs. untreated cells. SOD1(G93A) microglial cells were exposed to $1 \mu \mathrm{g} / \mathrm{ml}$ LPS and used for d, e qRT-PCR and Western blotting analysis of iNOS (d) and gp91 phox (e) at the indicated times. Gene expression values were normalized to Gapdh and presented as $2^{-\Delta \Delta \mathrm{Ct}}$. Relative mRNA gene abundance in untreated cells was assumed to be 1 (control). Data represent mean $\pm \mathrm{SD}(n=5) .{ }^{*} p<0.05$ vs. untreated cells. GAPDH was used as loading control in Western Blotting analyses. The images are representative of one out of three separate experiments; f detection of NO production after a 24-h LPS exposure by Griess Reagent ( $100 \%$ control NO absorbance, $0.29 \pm 0.05)$. ${ }^{*} p<0.05$ vs. untreated cells; $\mathbf{g}$ detection of ROS generation by DCF fluorescence after $24 \mathrm{~h}$ LPS exposure. Scale bar, $100 \mu \mathrm{m}$; h immunolocalization of nitrotyrosine. The immunofluorescence staining was performed as described using anti-nitrotyrosine antibody after a 6-h LPS exposure. The images are representative of one out of three separate experiments. Scale bar, $100 \mu \mathrm{m}$

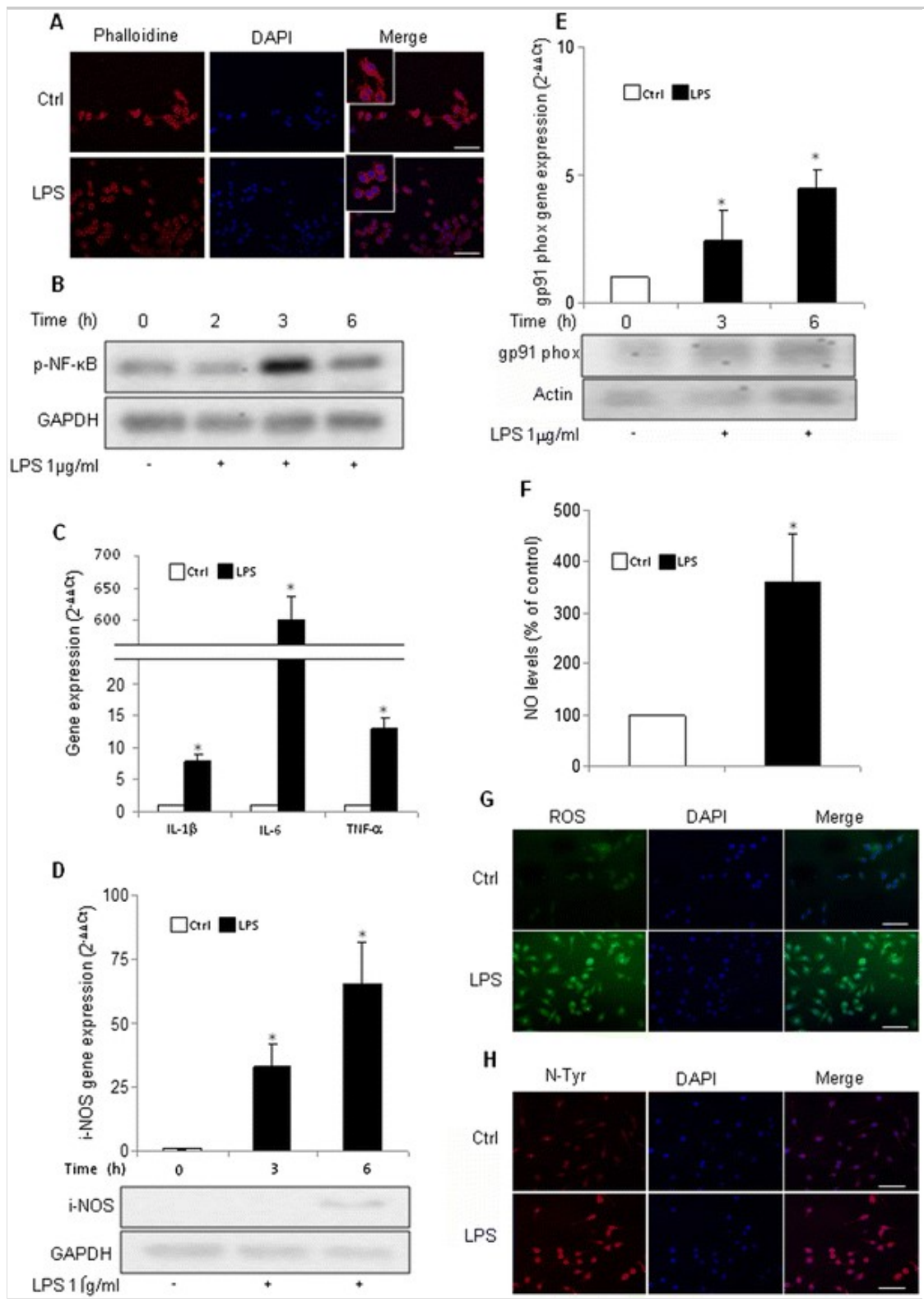

The activity of iNOS and NOX2 enzymes can be inferred by the observation that the concentration of their products increases after LPS exposure (Fig. 2f, g). Indeed, LPS induces about a 400\% increase in NO levels (Fig. 2f) and an increase in ROS levels, as determined by DCFH staining ( $240 \pm 135$ vs. 517,860 $\pm 56,541$ pixel over threshold) (Fig. $2 \mathrm{~g}$ ) and by dihydroethidium staining (Online Resource 1A). By reacting with NOX2-produced superoxide anion, NO can form highly reactive peroxynitrite, responsible for protein nitration [22]. We found that LPS treatment increases the levels of nitrotyrosine ( $150 \pm 58$ vs. $4440 \pm 1057$ pixel over threshold) (Fig. $2 \mathrm{~h})$ suggesting that LPS-exposed SOD1(G93A) microglial cells undergo an oxidative/nitrosative stress.

\section{Peroxynitrite Is Responsible for Inflammasome Activation}

It has been shown that peroxynitrite is involved in NLRP3 inflammasome activation in intracerebral hemorrhage 
(ICH)-induced inflammatory injury [23]. To test whether the same mechanism is responsible for LPS-induced NLRP3 activation in SOD1(G93A) microglial cells, we pre-treated the cells with specific iNOS and NOX2 inhibitors, i.e. 1400W and apocyanin, respectively. 1400W reduced the LPS-induced increase in NO levels (Fig. 3a) while it did not affect ROS levels (Online Resource 1B). Apocyanin reduced LPS-induced increase in ROS levels (3063 \pm 380 vs. $1030 \pm 118$ pixel over threshold) (Fig. 3b) and NO levels were also affected (Online Resource 1C). Both inhibitors reduced nitrotyrosine levels in LPS-treated SOD1(G93A) microglial cells (1400W - $172 \pm 28$ vs. $1400 \mathrm{~W}+\mathrm{LPS} 128 \pm 41$; APO $-329 \pm 72$ vs. APO + LPS - $356 \pm 94$ pixel over threshold) (Fig. $3 \mathrm{c}$ ). In the presence of each single inhibitor, caspase 1 activity is strongly reduced (Fig. 3d, e) indicating that LPS induces caspase1 activation in SOD1(G93A) microglial cells through peroxynitrite formation.

Fig. 3

Peroxynitrite is responsible for inflammasome activation. SOD1(G93A) microglial cells were exposed to $250 \mu \mathrm{M}$ $1400 \mathrm{~W}$ or $500 \mu \mathrm{M}$ APO prior to $1 \mu \mathrm{g} / \mathrm{ml}$ LPS used for a detection of NO production by Griess Reagent and $\mathbf{b}$ detection of ROS generation by DCF fluorescence (scale bar, $100 \mu \mathrm{m}$ ) after a 24-h LPS exposure. Absorbance of control cells $(0.29 \pm 0.05)$ was assumed as $100 \%$. Data represent mean $\pm \operatorname{SD}(n=3)$. ${ }^{*} p<0.05$ vs. control cells; ${ }^{*} p<0.05$ vs. LPS-treated cells; c immunolocalization of nitrotyrosine after a 6-h LPS exposure. Scale bar, $100 \mu \mathrm{m}$. SOD1(G93A) microglial cells, treated with d $250 \mu \mathrm{M} 1400 \mathrm{~W}$ or e $500 \mu \mathrm{M}$ APO prior to a $6-\mathrm{h} 1 \mu \mathrm{g} / \mathrm{ml} \mathrm{LPS}$ exposure, were collected and total extracts were subjected to Western blotting. SOD1(G93A) microglial cells were exposed to a 6-h $1 \mu \mathrm{g} / \mathrm{ml} \mathrm{LPS}$ and cell extracts were subjected to immunoprecipitation (f) with anti- nytrotyrosine antibody followed by Western Blotting analysis of NLRP3. The images are representative of one out of three separate experiments. GAPDH was used as loading control

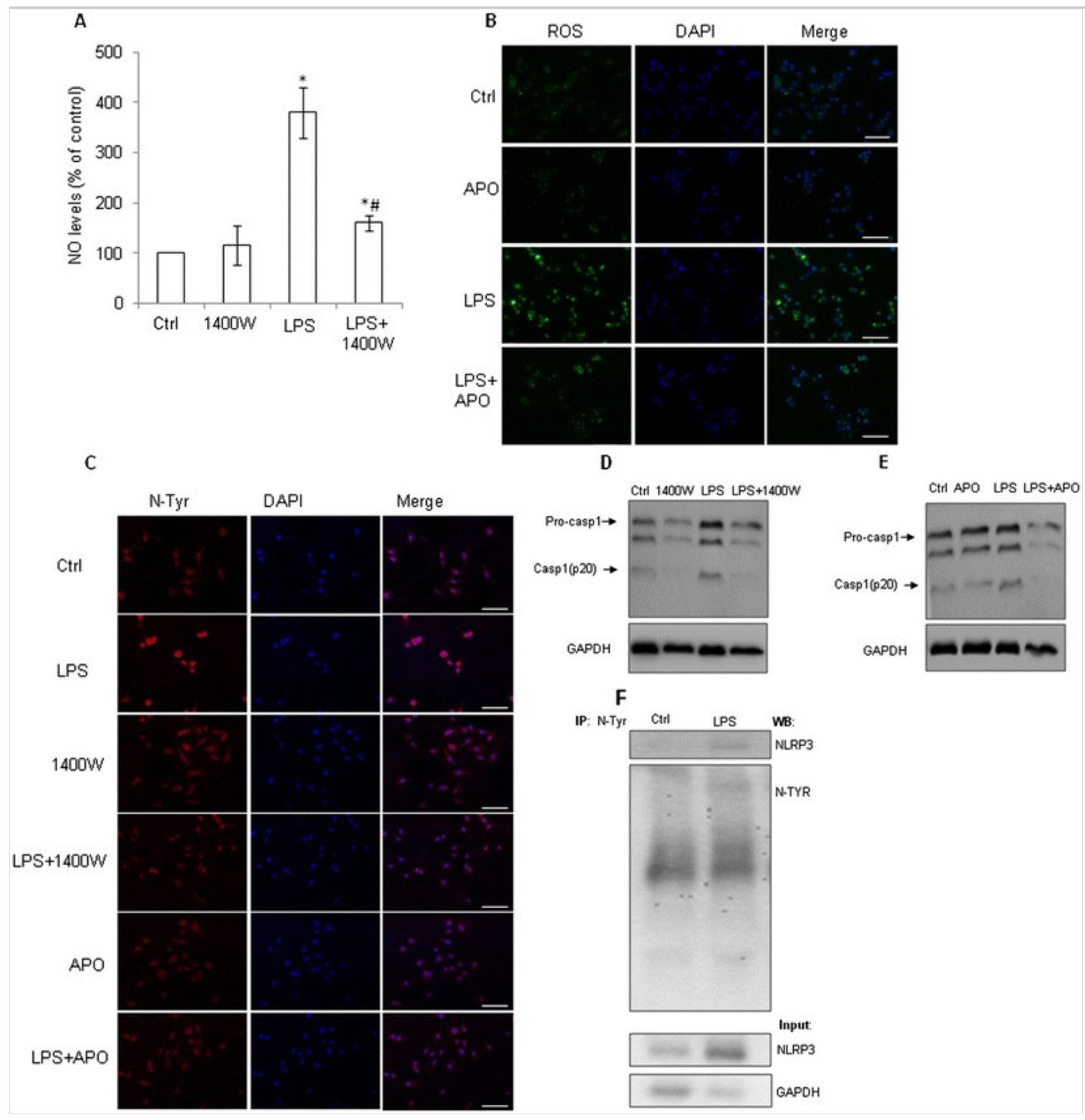


To determine whether peroxynitrite modifies the NLRP3 inflammasome, we performed immunoprecipitation to exploreNLRP3 nitration in SOD1(G93A) microglial cells treated with LPS for $6 \mathrm{~h}$ (Fig. 3f). We found that NLRP3 is immunoprecipitated by anti-nitrotyrosine antibody in LPS-treated cells suggesting that this post-translational modification, induced by the increase in peroxynitrite, could modify NLRP3 function in SOD1(G93A) microglial cells.

\section{Protein Nitration and Caspase 1 Activation Are Increased in the Spinal Cord of SOD1(G93A) Mice}

To determine whether spinal cords of pre-symptomatic hSOD1(G93A) mice display the molecular features of the mechanism described in vitro, we investigated caspase 1 activation and protein nitration in the gray matter of the lumbar spinal cord of pre-symptomatic (W13) hSOD1(G93A) mice. We found that the spinal cord of ALS mouse model is robustly stained by the anti-nitrotyrosine antibody ( $912 \pm 400$ vs. $14,878 \pm 1622$ pixel over threshold) (Fig. 4a) indicating that, even in the pre-symptomatic stage of the pathology, mice undergo a significant increase in peroxynitrite formation. Moreover, the spinal cord of pre-symptomatic mice shows a strong increase in active caspase1 (Fig. 4b). Image analysis of fluorescent signals indicate that caspase 1 fluorescence was approximately ten times higher in SOD1(G93A) compared with control spinal cord ( $280 \pm 148$ vs. $2478 \pm 504$ pixel over threshold). Moreover, the induction of caspase 1 activity was much greater in SOD1(G93A) spinal cord than in $5 \mathrm{mg} / \mathrm{kg}$ LPS-treated control spinal cords. These data indicate that peroxynitrite formation and inflammasome activation are early concomitant events in ALS pathology and can be, at least in part, responsible for motoneuron cell death.

\section{Fig. 4}

Protein nitration and caspase1 activation are increased in the spinal cord of SOD1(G93A) mice. Lumbar spinal cord of 13-week-old untreated (control), LPS-treated (5 mg/kg; LPS) and hSOD1(G93A) (pre-symptomatic) mice were used for immunolocalization of nytrotyrosine (a) and detection of activate caspase1 (b). The images are representative of one out of three separate experiments. Scale bar, $100 \mu \mathrm{m}$

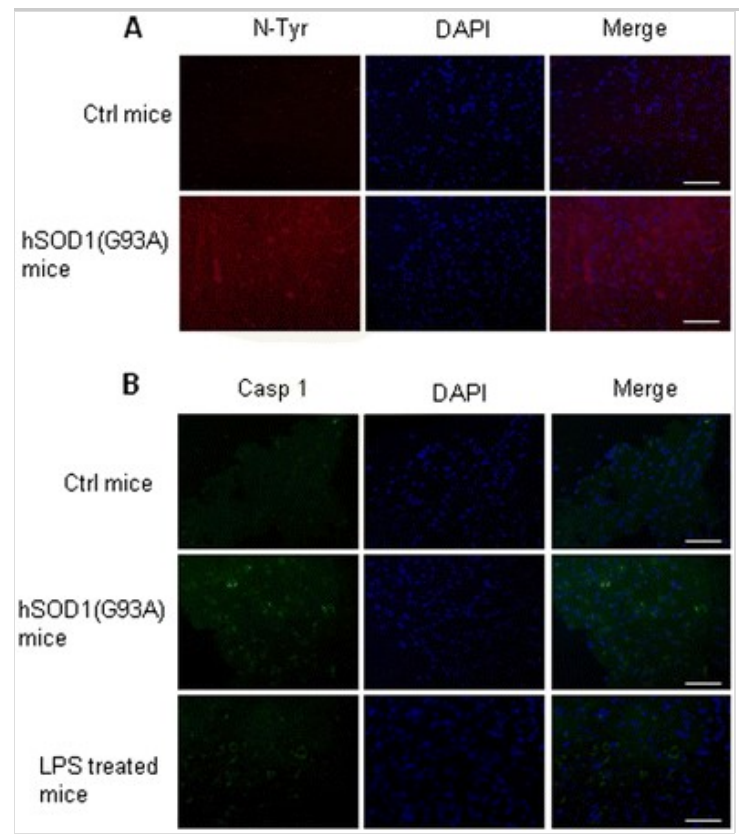

\section{Discussion}

Here we showed, for the first time, that that peroxynitrite activates caspase 1 and the downstream inflammatory cascade in SOD1(G93A) microglial cells thus contributing to one of the several ALS pathogenic mechanisms. To date, Feng et al. [23] showed that peroxynitrite is a downstream signaling molecule of P2X7 receptor, which plays a critical role in triggering NLRP3 inflammasome activation, but by IP we have shown that nitration occurs precisely at NLRP3 levels. Although the pathogenesis of ALS is believed to involve a vicious circle of inflammation leading to neurodegeneration and further inflammation, the mechanisms supporting this chronic inflammatory response remain poorly understood. In this study, we explored the link between protein nitration, resulting from an acute inflammatory stimulus (LPS), and the activation of the NLRP3 inflammasome as a possible driver of chronicity in the context of ALS. In glial cells expressing the (G93A) mutant SOD1, we demonstrated that challenge with LPS can 
drive protein nitration-dependent NLRP3 activation. Moreover, we showed that the L4-L6 region of the spinal cord of pre-symptomatic (W13) SOD1(G93A) mice undergoes massive protein nitration with a concomitant increase in caspase 1 activity, two events which could support our in vitro results. By connecting an acute inflammatory response to activation of the NLRP3 inflammasome (and thereby IL-1 $\beta$ production/secretion), these data together identify protein nitration as a potential 'smoking gun' in the search for molecular mechanisms underpinning the chronic neuroinflammation driving ALS pathogenesis.

Several pathogenic factors have been proposed to explain ALS pathogenic mechanisms [24]; these include increased production of reactive oxygen species (ROS) [25] and nitroxyradicals responsible for protein tyrosine nitration $[23,26,27,28]$. The increase in ROS and NO is a hallmark of neuroinflammation, i.e. the occurrence of a neuroinflammatory reaction consisting of activated glial cells, mainly microglia and astrocytes. Microglia, the resident mononuclear phagocytes in the brain, are activated to protect the neuronal environment in response to infection or damage. In these conditions, microglia acquire an amoeboid appearance, up-regulate oxidant molecules such as NO and superoxide anion and secrete pro-inflammatory molecules such as TNF $\alpha$ and IL-1 [6]. For these reasons, we used immortalized microglial cells from hSOD1(G93A) mice to study the mechanism leading to neuroinflammation. Thus we exposed SOD1(G93A) overexpressing cells to LPS, a common inflammogen able to induce neuroinflammation and reactive microgliosis in vivo and in vitro [21]. The exposure of SOD1(G93A) microglial cells to LPS induced an increase in gene and protein expression of iNOS and gp91phox subunit of NOX2, resulting in an increase in NO and ROS levels that consequently drove an increase in protein nitration. Several reports link oxidative/nitrosative stress to ALS. Indeed, it has been described that NOX2 produces damaging ROS in ALS [29, 30]; SOD1 mutations induce NADPH oxidase-dependent ROS production in microglia of SOD1(G93A) mice, leading to motor neuron death [31,32]; and the deletion of iNOS gene, significantly extends the lifespan of SOD1(G93A) mice [33]. These data suggest that protein nitration may be one of the underlying causes of the pathology.

It is worth noticing that, besides NOX2, mitochondria can also contribute to ROS generation. Indeed, in neurodegenerative disorders, an increase in mitochondrial ROS generation, leading to NLPR3 activation, has been reported $[34,35,36]$.

We found that SOD1(G93A) microglial cells, activated by LPS stimulation, increased gene expression and protein secretion of pro-inflammatory cytokines, including IL-1 $\beta$. The IL-1 family of cytokines plays a role in host defense and immune system regulation in inflammatory diseases [6]. IL-1 $\beta$ is produced as an inactive precursor (pro-IL-1 $\beta$ ) by microglia [6]. The biological effects of IL-1 $\beta$ follow the cleavage and activation of pro-IL- $1 \beta$. The mature form is then released and acts on the type I IL-1 receptor (IL-1RI) on the surface of responsive cells. Caspase1 is the key protease essential for the processing of pro-IL-1 $\beta$. The activity of caspase 1 is regulated by its recruitment to inflammasomes that follows an inflammatory stress [11]. NLRP3, the best-characterized inflammasome receptor $[10,11]$, upon activation by many disease-associated molecules, oligomerizes with the adaptor ASC and caspase 1 to form the NLRP3 inflammasome. It is conceivable that activated SOD1(G93A) microglial cells have inflammasome components to secrete mature IL-1 $\beta$. We observed that all inflammasome receptors are indeed expressed in the SOD1(G93A) microglial cell line and that LPS stimulation increased the gene and protein expression of NLRP3. By binding to TLR4, LPS, via NF- $\kappa B$, induces the expression of NLRP3 [10]. Moreover, in dendritic cells, TLR4 signaling is sufficient to trigger inflammasome activation [10,37]. These reports suggest that TLR4 ligands are active players in modulating inflammasome activation and that, depending on cell type, they can interact with cell-specific target molecules and lead to an active inflammasome, even in the absence of other stimuli. Activated SOD1(G93A) microglial cells also showed an active caspase1, indicating that all the steps needed to obtain a mature IL- $1 \beta$ are present and active in this cell line. Indeed, LPS exposure caused an increase in the mature form of IL-1 $\beta$. Recently, the capacity to form a functional NLRP3 inflammasome and to secrete IL-1 $\beta$, has been attributed to the microglial compartment in the mouse brain [38]. On the other hand, NLRP3, ASC, active caspase1 and IL-18 are concomitantly expressed in astroglial cells of spinal cord tissue samples from SOD1(G93A) mice and in sporadic ALS patients [14].

Our work confirmed the presence of active caspase 1 in spinal cord of SOD1(G93A) mice [14, 39] and causally linked nitrotyrosyne levels to the activation of caspase1. Indeed, when the levels of nitrated proteins were reduced by specific inhibition of iNOS and NOX2, the levels of active caspase1 were also reduced. It has been shown that iNOS-positive activated glial cells were increased in transgenic SOD1(G93A) mice and that the reduction in the levels of NO by L-arginine administration, reduced motor neuron death and glial activation [40]. Moreover, the pharmacological inhibition of NF- $\mathrm{KB}$ activation and iNOS expression, extended lifespan of transgenic mouse model 
of ALS [41]. Furthermore, genetic deletion and specific inhibition of NOX2 in the SOD1(G93A) mice was reported to remarkably increase survival [29].

These results underpin that the increased levels of NO and ROS are potentially responsible for ALS pathogenesis and/or progression. We propose that the noxious effects of oxidative/nitrosative stress are due to inflammasome activation possibly due to nitration of NLRP3 itself. This hypothesis is in line with the results obtained in intracelebral hemorrage where $\mathrm{P} 2 \mathrm{X} 7$ receptor, which has peroxynitrite as a downstream signaling molecule, plays a critical role in NLRP3 inflammasome activation [23]. It is to note that P2X7 receptor is involved in NLRP3 inflammasome activation [42]. Moreover, a blood-brain barrier permeable P2X7 receptor antagonist significantly increased motor neuron survival and decreased lumbar spinal cord microgliosis, delayed the onset of the disease and improved the motor performance of ALS mice. These effects have been related to the modulation of inflammatory markers such as NF- $\kappa$ B, NOX2 and IL-1 $\beta[43]$.

\section{Conclusion}

Our results indicate that NOX2/iNOS-dependent formation of peroxynitrite, a downstream signaling component of reactive microgliosis, may be a key trigger of caspase 1 activation (Fig. 5). Thus, inhibition of peroxynitrite formation could be a potential therapeutic target for ALS.

\section{Fig. 5}

Schematic representation of peroxynitrite-induced inflammasome cascade activation. Neuroinflammatory stimuli, through NF- $\kappa$ B activation, induce in glial cells the expression of iNOS and gp91 phox subunit of NOX2 resulting in increased levels of ROS and NO [44]. These species spontaneously react to form peroxynitrite, which, in turn, affect inflammasome activation. This event leads to the cleavage of pro- to mature IL- $1 \beta$, which upsurges the vicious circle of inflammation

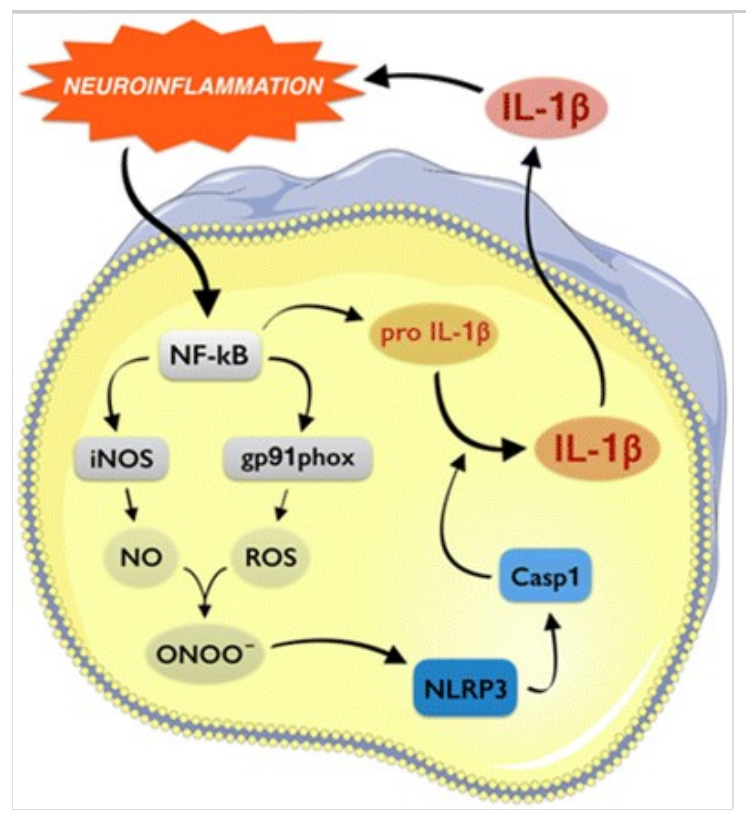

\section{Acknowledgements}

We thank Dr. G. Pietrini (Università di Milano) for providing immortalized SOD1(G93A) microglial cells. This study was supported by FIRB 'Futuro in ricerca' grant funded by MIUR (RBFR12BUMH) and Fondazione Cassa di Risparmio di Perugia (2015.0326.021). The funders had no role in the study design, data collection and analysis, decision to publish or preparation of the manuscript.

\section{Electronic Supplementary Material}

\section{ESM 1}

(DOCX $12 \mathrm{~kb})$. 
Fig. S1

(GIF $205 \mathrm{~kb})$.

High-resolution image (TIFF $1993 \mathrm{~kb}$ ).

\section{References}

1. Kiernan MC, Vucic S, Cheah BC, Turner MR, Eisen A, Hardiman O, Burrell JR, Zoing MC (2011)

Amyotrophic lateral sclerosis. Lancet 377:942-955

2. Rosen DR, Siddique T, Patterson D, Figlewicz DA, Sapp P, Hentati A, Donaldson D, Goto J et al (1993) Mutations in $\mathrm{Cu} / \mathrm{Zn}$ superoxide dismutase gene are associated with familial amyotrophic lateral sclerosis. Nature 362:59-62

3. Clement AM, Nguyen MD, Roberts EA, Garcia ML, Boillée S, Rule M, McMahon AP, Doucette W et al (2003) Wild-type nonneuronal cells extend survival of SOD1 mutant motor neurons in ALS mice. Science 302:113-117

4. Boillee S, Yamanaka K, Lobsiger CS, Copeland NG, Jenkins NA, Kassiotis G, Kollias G, Cleveland DW (2006) Onset and progression in inherited ALS determined by motor neurons and microglia. Science 312:1389-1392

5. Lee J, Hyeon SJ, Im H, Ryu H, Kim Y, Ryu H (2016) Astrocytes and microglia as non-cell autonomous players in the pathogenesis of ALS. Exp Neurobiol 25:233-240

6. Philips T, Robberecht W (2011) Neuroinflammation in amyotrophic lateral sclerosis: role of glial activation in motor neuron disease. Lancet Neurol 10:253-263

7. Alexianu ME, Kozovska M, Appel SH (2001) Immune reactivity in a mouse model of familial ALS correlates with disease progression. Neurology 57:1282-1289

8. Sargsyan SA, Blackburn DJ, Barber SC, Grosskreutz J, De Vos KJ, Monk PN, Shaw PJ (2011) A comparison of in vitro properties of resting SOD1 transgenic microglia reveals evidence of reduced neuroprotective function. BMC Neurosci 12:91

9. Meissner F, Molawi K, Zychlinsky A (2010) Mutant superoxide dismutase 1-induced IL-1beta accelerates ALS pathogenesis. Proc Natl Acad Sci U S A 107:13046-13050

10. Jo EK, Kim JK, Shin DM, Sasakawa C (2016) Molecular mechanisms regulating NLRP3 inflammasome activation. Cell Mol Immunol 13:148-159

11. Schroder K, Tschopp J (2010) The inflammasomes. Cell 140:821-832

12. Zhao W, Beers DR, Henkel JS, Zhang W, Urushitani M, Julien JP, Appel SH (2010) Extracellular mutant SOD1 induces microglial-mediated motoneuron injury. Glia 58:231-243

13. Volpe CM, Nogueira-Machado JA (2015) Is innate immunity and Inflammasomes involved in pathogenesis of amyotrophic lateral sclerosis (ALS)? Recent Pat Endocr Metab Immune Drug Discov 9:40-45

14. Johann S, Heitzer M, Kanagaratnam M, Goswami A, Rizo T, Weis J, Troost D, Beyer C (2015) NLRP3 inflammasome is expressed by astrocytes in the SOD1 mouse model of ALS and in human sporadic ALS patients. Glia 63:2260-2273 
15. Zhao W, Beers DR, Bell S, Wang J, Wen S, Baloh RH, Appel SH (2015) TDP-43 activates microglia through NF- $\kappa$ B and NLRP3 inflammasome. Exp Neurol 273:24-35

16. Righi M, Pierani A, Boglia A, De Libero G, Mori L, Marini V, Ricciardi-Castagnoli P (1989) Generation of new oncogenic murine retroviruses by cotransfection of cloned AKR and MH2 proviruses. Oncogene: 223-230

17. Minelli A, Grottelli S, Mierla A, Pinnen F, Cacciatore I, Bellezza I (2012) Cyclo(His-Pro) exerts anti-inflammatory effects by modulating NF- $\kappa$ B and Nrf2 signalling. Int J Biochem Cell Biol 44:525-535

18. Eruslanov E, Kusmartsev S (2010) Identification of ROS using oxidized DCFDA and flow-cytometry. Methods Mol Biol 594:57-72

19. Bellezza I, Grottelli S, Gatticchi L, Mierla AL, Minelli A (2014) $\alpha$-Tocopheryl succinate pre-treatment attenuates quinone toxicity in prostate cancer PC3 cells. Gene 539:1-7

20. Kawanokuchi J, Shimizu K, Nitta A, Yamada K, Mizuno T, Takeuchi H, Suzumura A (2008) Production and functions of IL-17 in microglia. J Neuroimmunol 194:54-61

21. Bellezza I, Grottelli S, Mierla AL, Cacciatore I, Fornasari E, Roscini L, Cardinali G, Minelli A (2014) Neuroinflammation and endoplasmic reticulum stress are coregulated by cyclo(His-Pro) to prevent LPS neurotoxicity. Int J Biochem Cell Biol 51:159-169

22. Ferrer-Sueta G, Radi R (2009) Chemical biology of peroxynitrite: kinetics, diffusion, and radicals. ACS Chem Biol 4:161-177

23. Feng L, Chen Y, Ding R, Fu Z, Yang S, Deng X, Zeng J (2015) P2X7R blockade prevents NLRP3 inflammasome activation and brain injury in a rat model of intracerebral hemorrhage: involvement of peroxynitrite. J Neuroinflammation 12:190

24. Bruijn LI, Miller TM, Cleveland DW (2004) Unraveling the mechanisms involved in motor neuron degeneration in ALS. Annu Rev Neurosci 27:723-749

25. Barber SC, Shaw PJ (2010) Oxidative stress in ALS: key role in motor neuron injury and therapeutic target. Free Radic Biol Med 48:629-641

26. Beckman JS, Carson M, Smith CD, Koppenol WH (1993) ALS, SOD and peroxynitrite. Nature 364:584

27. Pacher P, Beckman JS, Liaudet L (2007) Nitric oxide and peroxynitrite in health and disease. Physiol Rev $87: 315-424$

28. Heneka MT, Kummer MP, Latz E (2014) Innate immune activation in neurodegenerative disease. Nat Rev Immunol 14:463-477

29. Harraz MM, Marden JJ, Zhou W, Zhang Y, Williams A, Sharov VS, Nelson K, Luo M et al (2008) SOD1 mutations disrupt redox-sensitive Rac regulation of NADPH oxidase in a familial ALS model. J Clin Invest 118:659-670

30. Li Q, Spencer NY, Pantazis NJ, Engelhardt JF (2011) Alsin and SOD1(G93A) proteins regulate endosomal reactive oxygen species production by glial cells and proinflammatory pathways responsible for neurotoxicity. $\mathrm{J}$ Biol Chem 286:40151-40162

31. Marden JJ, Harraz MM, Williams AJ, Nelson K, Lu M, Paulson H, Engelhard JF (2007) Redox modifier genes in amyotrophic lateral sclerosis in mice. J Clin Invest 117:2913-2919

32. Wu DC, Ré DB, Nagai M, Ischiropoulos H, Przedborski S (2006) The inflammatory NADPH oxidase enzyme modulates motor neuron degeneration in amyotrophic lateral sclerosis mice. Proc Natl Acad Sci U S A

103:12132-12137 
33. Martin LJ, Liu Z, Chen K, Price AC, Pan Y, Swaby JA, Golden WC (2007) Motor neuron degeneration in amyotrophic lateral sclerosis mutant superoxide dismutase-1 transgenic mice: mechanisms of mitochondriopathy and cell death. J Comp Neurol 500:20-46

34. Sandhir R, Halder A, Sunkaria A (2016) Mitochondria as a centrally positioned hub in the innate immune response. Biochim Biophys Acta. doi: 10.1016/j.bbadis.2016.10.020

35. Won JH, Park S, Hong S, Son S, Yu JW (2015) Rotenone-induced impairment of mitochondrial electron transport chain confers a selective priming signal for NLRP3 inflammasome activation. J Biol Chem 290:27425-27437

36. Chen L, Na R, Boldt E, Ran Q (2015) NLRP3 inflammasome activation by mitochondrial reactive oxygen species plays a key role in long-term cognitive impairment induced by paraquat exposure. Neurobiol Aging 36:2533-2543

37. He Y, Franchi L, Núñez G (2013) TLR agonists stimulate Nlrp3-dependent IL-1 $\beta$ production independently of the purinergic P2X7 receptor in dendritic cells and in vivo. J Immunol 190:334-339

38. Gustin A, Kirchmeyer M, Koncina E, Felten P, Losciuto S, Heurtaux T, Tardivel A, Heuschling P et al (2015) NLRP3 inflammasome is expressed and functional in mouse brain microglia but not in astrocytes. PLoS One 10:e0130624

39. Li M, Ona VO, Guégan C, Chen M, Jackson-Lewis V, Andrews LJ, Olszewski AJ, Stieg PE et al (2000) Functional role of caspase-1 and caspase-3 in an ALS transgenic mouse model. Science 288:335-339

40. Lee J, Ryu H, Kowall NW (2009) Differential regulation of neuronal and inducible nitric oxide synthase (NOS) in the spinal cord of mutant SOD1 (G93A) ALS mice. Biochem Biophys Res Commun 387:202-206

41. Xu Z, Chen S, Li X, Luo G, Li L, Le W (2006) Neuroprotective effects of (-)-epigallocatechin-3-gallate in a transgenic mouse model of amyotrophic lateral sclerosis. Neurochem Res 31:1263-1269

42. Dubyak GR (2012) P2X7 receptor regulation of non-classical secretion from immune effector cells. Cell Microbiol 14:1697-1706

43. Apolloni S, Amadio S, Parisi C, Matteucci A, Potenza RL, Armida M, Popoli P, D’Ambrosi N et al (2014) Spinal cord pathology is ameliorated by P2X7 antagonism in a SOD1-mutant mouse model of amyotrophic lateral sclerosis. Dis Model Mech 7:1101-1109

44. Brigelius-Flohé R, Flohé L (2011) Basic principles and emerging concepts in the redox control of transcription factors. Antioxid Redox Signal 15:35-81 